\title{
PREDICCIONES DE LA ENTROPÍA PROPORCIONAL EN LA DINÁMICA CARDÍACA
}

\author{
JAVIER RODRÍGUEZ ${ }^{1 *}$, SIGNED PRIETO², CATALINA CORREA ${ }^{3}$, YOLANDA SORACIPA ${ }^{4}$, JESSICA MORA ${ }^{5}$, \\ PEDRO BERNAL ${ }^{6}$, MARÍA FORERO', \\ CAMILO ACUÑÄ.
}
${ }^{1}$ MD. Director del Grupo Insight. Director de la Línea de Profundización e Internado Especial Física y Matemáticas Aplicadas a la Medicina, Universidad Militar Nueva Granada. Centro de Investigaciones Clínica del Country. Bogotá, Colombia.
${ }^{2}$ Investigadora Grupo Insight - Universidad Militar Nueva Granada.
Centro de Investigaciones Clínica del Country. Bogotá, Colombia.
${ }^{3}$ Psic. Investigadora Grupo Insight. Docente línea de Profundización e Internado Especial: Física y Matemáticas Aplicadas a la Medicina, Universidad Militar Nueva Granada.
Centro de Investigaciones Clínica del Country. Bogotá, Colombia.
${ }^{4}$ Lic Fis. Investigadora Grupo Insight - Universidad Militar Nueva Granada.
Centro de Investigaciones Clínica del Country. Bogotá, Colombia.
${ }^{5}$ Internado Especial: Física y Matemáticas Aplicadas a la Medicina, Universidad Militar Nueva Granada. Bogotá, Colombia.
${ }^{6}$ Ing. Sistemas. Bogotá, Colombia.
${ }^{7}$ MD. Investigadora Grupo Insight. Bogotá, Colombia.
${ }^{8}$ Fís. Universidad de los Andes. Investigador Grupo Insight. Bogotá, Colombia.
"Correspondencia: Javier Rodríguez. E-mail: grupoinsight2025@yahoo.es

Recibido: Enero 7 de 2015 Aceptado: Marzo 31 de 2015

\begin{abstract}
Resumen
Antecedentes: A partir de la teoría de sistemas dinámicos se desarrolló una metodología que permite diferenciar dinámicas cardíacas normales, en distintos niveles de enfermedad y en evolución entre estos estados. Esto se hizo cuantificando en el espacio de fases, mediante la entropía y sus proporciones, la probabilidad de parejas ordenadas de frecuencias cardiacas.

Objetivo: Aplicar clínicamente la metodología desarrollada a 400 dinámicas cardíacas para establecer su efectividad comparándola con el diagnóstico clínico convencional.
\end{abstract}


Método: Se tomaron Holters de 400 individuos; 50 diagnosticados como normales y 350 con diferentes patologías. Para cada uno se construyó un atractor en el mapa de retardo y se evaluó, mediante la entropía y sus proporciones, la probabilidad de ocupación de pares ordenados de frecuencias cardíacas durante 18 horas. Se compararon las medidas obtenidas con los valores de normalidad y enfermedad establecidos previamente para obtener el diagnóstico de cada Holter. Las conclusiones del Holter y los antecedentes clínicos sólo fueron desenmascarados luego de haber aplicado la metodología físico-matemática para calcular sensibilidad, especificidad y coeficiente Kappa respecto al Gold-Estándar.

Resultados: con las proporciones de la entropía de los atractores se diferenciaron dinámicas cardíacas agudas, crónicas, normales, y evolución normalidad-enfermedad. Se confirmó la aplicabilidad clínica de la metodología predictiva desarrollada para el Holter, que mostró una sensibilidad y especificidad del 100\%. La concordancia entre el Gold-Estándar y el diagnóstico físicomatemático fue 1.

Conclusiones: La aplicación de la metodología permitió establecer cuantitativamente estados de normalidad y enfermedad de la dinámica cardíaca, evidenciando una auto-organización del atractor dinámico geométrico que constituye un método de ayuda diagnóstica aplicable a la clínica.

Palabras Clave: diagnóstico, enfermedades cardiovasculares, adulto, frecuencia cardíaca, dinámicas no Lineales.

\title{
PREDICTIONS OF PROPORTIONAL ENTROPY IN CARDIAC DYNAMICS
}

\begin{abstract}
Based on dynamic systems theory, a methodology that allows to differentiate normal cardiac dynamics, different levels of abnormality and evolution between these states, was developed. This was done by quantifying the probability of ordered pairs of heart rates in the phase space, through entropy and its proportions. To apply in a clinical setting the developed methodology to 400 cardiac dynamics in order to establish its effectiveness by comparing it with the conventional diagnosis. Holters from 400 individuals were tested for a minimum of 18 hours; 50 with normal diagnosis and 350 with different pathologies. An attractor was built for each one of them in the delay map, and the occupation probability of ordered pairs of heart rates was evaluated through entropy and its proportions. Afterwards, results were compared with values of normality and disease previously established to obtain the diagnosis for each Holter. The findings of Holter and medical history were only revealed after applying the physical-mathematical methodology, in order to calculate sensitivity, specificity and Kappa coefficient regarding to the Gold-Standard. With the entropy proportions of the attractors, acute cardiac dynamics were differentiated from chronic and normal ones, as well as the evolution between normality and disease. A clinical application of the predictive methodology for Holter was developed. Sensitivity and specificity were both $100 \%$ and the correlation between the Gold-Standard and the physical-mathematical diagnosis was 1 . The application of the methodology allowed establishing quantitatively states of normality and disease of the cardiac dynamic, showing a self-organization of the geometrical dynamic attractor.
\end{abstract}

Keywords: diagnosis, cardiovascular diseases, adult, heart rate, nonlinear dynamics. 


\title{
PREVISÕES DE ENTROPIA PROPORCIONAL NA DINÂMICA CARDÍACA
}

\begin{abstract}
Resumo
Antecedentes: A partir da teoria de sistemas dinâmicos se desarrolho uma metodologia que permite diferenciar dinâmicas cardíacas normais, em distintos niveles de doença e na evolução entre estes estados, o qual foi realizado quantificando no espaço de fases a probabilidade de pares ordenados de frequências cardíacas, através da entropia e as suas proporções.

Objetivo: Aplicar clinicamente a metodologia desenvolvida a 400 dinâmicas cardíacas para estabelecer sua eficácia comparada com o diagnóstico clínico convencional.

Método: Se tiraram Holters de 400 pessoas, 50 deles foram diagnosticados como normais e 350 têm diferentes patologias. Um atractor no mapa de retardos foi construído para cada um deles e, através da entropia $e$ as suas proporções, a probabilidades de ocupação de pares ordenados de frequências cardíacas durante 18 horas foi avaliada. Além disso, as medidas obtidas foram comparadas com os valores de normalidade e doenças estabelecidas previamente para obter $\mathrm{o}$ diagnostico de cada Holter. As conclusões do Holter e os antecedentes clínicos somente foram descobertos logo de aplicar a metodologia físico-matemática para calcular a sensibilidade, especificidade y coeficiente Kappa acerca do Padrão-Ouro (Gold-Standard).

Resultados: As dinâmicas cardíacas agudas, crônicas, normais y a evolução da normalidade e as doenças foram diferenciadas pelas proporções da entropia dos atractores. Confirmou-se a aplicabilidade clínica da metodologia preditiva desenvolvida para o Holter, a qual demonstrou uma sensibilidade e especificidade de 100\%. O acordo entre o Padrão-Ouro (Gold-Standard) e o diagnóstico físico-matemático foi 1.

Conclusões: A aplicabilidade da metodologia permitiu estabelecer quantitativamente estado de normalidade e doenças da dinâmica cardíaca, mostrando uma auto-organização do atrator dinâmico geométrico que constitui um método de ajuda diagnostica aplicável à clínica.
\end{abstract}

Palavras-chave: Diagnóstico, doenças cardiovasculares, adultos, frequências cardíacas, dinâmicas não lineares. 


\section{Introducción}

La principal causa de muerte en el mundo son las enfermedades cardiovasculares, siendo 30\% la tasa de mortalidad por esta causa (1). El Departamento Administrativo Nacional de Estadística DANE reportó para el año 2009 un total de 43.205 casos de muerte por dos enfermedades cardíacas que se encuentran entre las cinco principales causas de mortalidad en Colombia (2), por lo que es necesario el desarrollo de metodologías efectivas de predicción de la dinámica cardíaca.

En la teoría de los sistemas dinámicos se pueden observar tres diferentes clases de atractores: el punto, el ciclo y el caótico (3): los dos primeros corresponden a dinámicas predecibles y el último se relaciona con una dinámica impredecible. Estos atractores presentan una forma irregular, denominándose fractales, los cuales a su vez se pueden dividir en tres clases: abstractos (4,5), salvajes (5), y estadísticos $(6,7)$. Para caracterizar la irregularidad de los atractores caóticos (8) generalmente se utiliza la dimensión fractal. Las medidas fractales han permitido la cuantificación y evaluación de diferentes sistemas morfológicos y fisiológicos (9-11).

La dinámica cardíaca presenta un comportamiento irregular, por lo que teorías físicas y matemáticas se han aplicado en el desarrollo de diferentes metodologías para la predicción de su comportamiento (12-23), sin embargo, la mayoría de los trabajos realizados hasta el momento requieren estudios posteriores para su aplicación clínica $(24,25)$. En contraposición, a partir de la teoría de los sistemas dinámicos, Rodríguez y col., han estudiado diferentes sistemas como la dinámica cardíaca fetal (26) y la de adultos (27-29), desarrollando una metodología de aplicación clínica para el Holter a partir de la evaluación de la entropía de la probabilidad de ocupación de parejas ordenadas de frecuencias cardiacas en el espacio de fases. Esta metodología ha permitido diferenciar dinámicas agudas, crónicas y normales (29), y ha mostrado ser útil en la predicción de la evolución de la dinámica cardíaca en la unidad de cuidados coronarios (30).

La experiencia clínica ha mostrado que el Holter es una de las herramientas más efectivas en el diagnóstico y valoración de arritmias cardíacas sintomáticas y asintomáticas, en la evaluación del riesgo de varias poblaciones cardiacas y en la valoración de la respuesta al tratamiento. El Holter es una prueba de electrocardiografía ambulatoria, de tipo no invasivo, que evalúa 2 o 3 canales electrocardiográficos durante un período de 24 a 48 horas, permitiendo determinar cambios dinámicos de los fenómenos eléctricos que son de corta duración y transitorios (31).
La teoría de la probabilidad cuantifica la ocurrencia futura de los posibles eventos de un experimento, para esto se define el espacio muestral que contiene a todos los eventos $(32,33)$. La termodinámica y la mecánica estadística estudian fenómenos en los que están involucrados un número de cuerpos del orden de $10^{23}$, constituyendo la primera de las tres revoluciones científicas de la física moderna, junto con la mecánica cuántica y los sistemas dinámicos (34). Para la descripción de estos fenómenos se utiliza el concepto de entropía, el cual fue enunciado inicialmente por Carnot y ha sido reinterpretado en la teoría cinética de los gases, la mecánica estadística y la teoría de la información $(35,36)$.

El propósito de la presente investigación es la aplicación de la metodología desarrollada previamente (29) a Holters con diferentes tipos de patología, para comprobar la capacidad predictiva de la metodología respecto al diagnóstico clínico convencional del Holter.

\section{Definiciones}

Mapa de retardo: Tipo de atractor que representa la relación entre un punto dado de una serie de tiempo graficado en el eje $\mathrm{x}$, y otro punto en la serie de tiempo graficada en el eje y (37). En este caso la serie de tiempo son las frecuencias cardiacas consecutivas en el tiempo.

Par ordenado de frecuencias cardiacas: Pareja de frecuencias cardiacas consecutivas del mapa de retardo representadas como $(\mathrm{X}, \mathrm{Y})$, se ubicarán en el rango de a cinco correspondiente de acuerdo a sus coordenadas.

Probabilidad de pares ordenados consecutivos en rangos de 5: Cociente entre el número de pares ordenados que ocupen un rango y el total de pares ordenados de todo el examen (33).

$P(X, Y)=\frac{\text { Número de pares ordenados en } X, Y}{\text { Total de pares ordenados en el trazado }}$ Ecuación 1

Donde: Xy Y son múltiplos de 5.

Entropía del atractor: La entropía (S) de la ocupación de un atractor en el espacio de fases se define como la suma de los productos de las probabilidades de los rangos (X, Y) (ver definición anterior), con sus correspondientes logaritmos:

$$
S=k \sum_{x=1}^{n} \sum_{y=1}^{n} P(X, Y) \times \operatorname{Ln} P(X, Y) \text { Ecuación } 2
$$

Donde: $P(X, Y)$ es la probabilidad para el rango $(\mathrm{X}, \mathrm{Y})$, con $\mathrm{X}$ 
y Y siendo múltiplos de $5, k$ la constante de Boltzmann $(34,36)$ y n es el número total de frecuencias del trazado.

Proporción S/k del atractor: Se obtiene al tomar la ecuación anterior y dividirla por la constante de Boltzmann $(k)$ :

$$
\frac{S}{k}=\sum_{x=1}^{n} \sum_{y=1}^{n} P(X, Y) \times \operatorname{Ln} P(X, Y) \text { Ecuación3 }
$$

Ahora, si agrupamos términos y tomamos los rangos (X,Y) cuya frecuencia de aparición sea de unidades, decenas, centenas y miles, llamamos:

$$
\mathrm{U}=\sum_{b} \sum_{a} P(a, b) x \operatorname{Ln} P(a, b)
$$

Donde: $(a, b)$ son los rangos con una frecuencia de aparición de unidades con a y b siendo múltiplos de 5 .

De igual manera se definen para:

$$
\begin{aligned}
& \text { Decenas (D): } \boldsymbol{D}=\sum_{d} \sum_{c} P(c, d) \times \operatorname{Ln} P(c, d) \\
& \text { Centenas C: } \boldsymbol{C}=\sum_{f} \sum_{e} P(e, f) \times \operatorname{Ln} P(e, f) \\
& \text { Y para miles (M): } \boldsymbol{M}=\sum_{h} \sum_{g} P(g, h) x \operatorname{Ln} P(g, h)
\end{aligned}
$$

Así pues la ecuación 2 tomará la forma:

$$
\frac{S}{k}=U+D+C+M=T \text { Ecuación } 4
$$

Proporciones de la entropía: Proporciones entre las partes y la totalidad de la ecuación 4 , así pues se definen como:

$$
U / T, D / T, C / T, M / T, C / M \text { y } D / T \quad \text { Ecuación } 5
$$

Regiones del atractor: Se definieron tres regiones para el atractor (29).

Región 1: Comprende todos los rangos de la frecuencia cardíaca comunes a Holter normales.

Región 2: Comprende la totalidad de los rangos ocupados por Holter normales, excluyendo los de la región 1.

Región 3: La región restante total del mapa de retardo, es decir, los rangos de la frecuencia cardíaca que no son ocupados por los prototipos normales.

\section{Materiales y métodos}

Se analizaron 400 Holters evaluados desde los parámetros convencionales por un cardiólogo experto, tomados de investigaciones previas realizadas por el Grupo Insight; 350 de éstos fueron diagnosticados con alguna patología (Tabla 1), y 50 fueron considerados normales.

Tabla 1. Consideraciones clínicas desde los parámetros convencionales para 28 de los Holter estudiados.

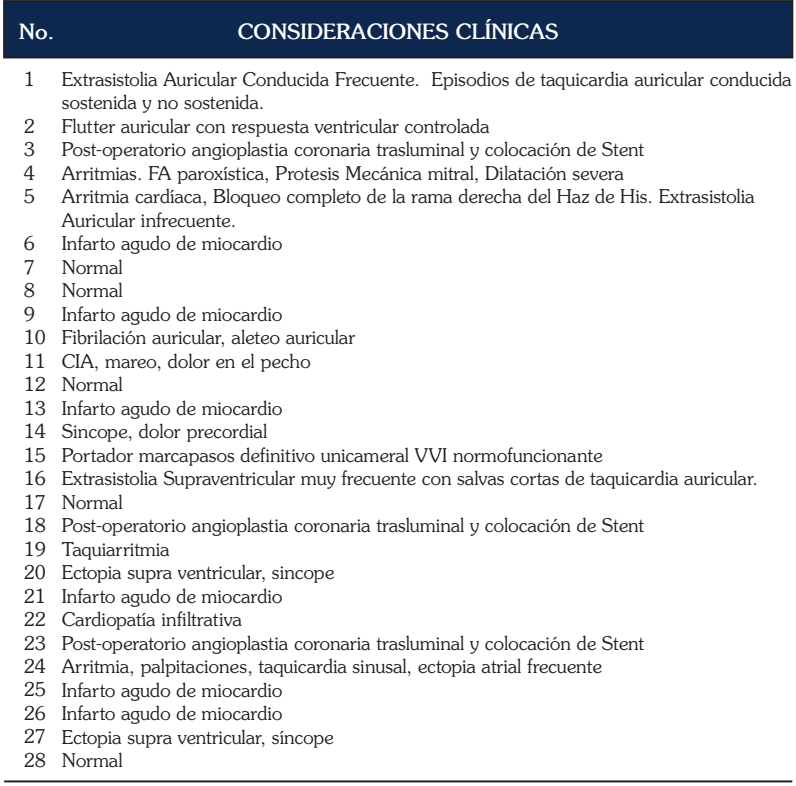

La información clínica y conclusiones registrados cada Holter fueron enmascarados. La única información no enmascarada correspondió a los valores para cada hora de frecuencias cardíacas mínimas y máximas y el número total de latidos, con base en los cuales se realizó una simulación de la totalidad de la dinámica para la obtención del diagnóstico físico-matemático. Luego se construyó un atractor en el mapa de retardo de acuerdo con la metodología desarrollada previamente para Holters de adultos (29), donde se graficó la frecuencia de aparición de pares ordenados de frecuencias. Posteriormente se evaluó para cada examen, en cada una de las regiones (ver definiciones), la probabilidad de ocupación respecto a la totalidad. Dicha probabilidad se cuantificó para cada uno de los rangos de a cinco en el espacio de fases (Ecuación 1), donde cada pareja de frecuencias cardiacas se consideró como un evento, y luego se evaluó la entropía de cada atractor (Ecuación 2).

Por último, se calculó la relación $\mathrm{S} / k$ (ver definiciones, ecuación 3) y se agruparon los sumandos que corresponden a probabilidades asociadas a frecuencias de ocupación de unidades (de 1 a 9), decenas (de 10 a 99), centenas (de 100 a 999) y miles (de 1000 a 9999) (Ecuación 4). Finalmente se 
evaluaron las proporciones de la entropía (ver definiciones, ecuación 5) para cada región determinada.

Aplicando la metodología diagnóstica desarrollada, se evaluó el criterio para diferenciar anormalidad y normalidad para cada Holter. Para esto se analizó el número de proporciones, en cualquiera de las tres regiones, que se encontrara fuera de los límites de normalidad definidos en trabajos previos (29). Se estableció entonces que si el número es dos o más la dinámica es anormal, de lo contrario la dinámica es normal (29).

Para cuantificar la evolución entre normalidad y anormalidad de cada dinámica, es decir, cuantificar específicamente la diferencia de la dinámica respecto a la normalidad, se realizaron restas entre los valores de las proporciones de la dinámica específica y los valores de los extremos de los intervalos de normalidad. Así, si el valor era mayor a los límites de normalidad se le restaba el límite superior de normalidad, mientras que los valores inferiores al valor mínimo de normalidad fueron restados de dicho valor límite. Finalmente, estas restas se sumaron de acuerdo a las frecuencias de aparición asociadas a las proporciones que se evaluaron, así pues $\mathbf{M}$ agrupó las restas de las proporciones asociadas a frecuencias de aparición de miles, $\mathrm{C}$ a las de centenas, $\mathrm{D}$ a las de decenas y $\mathrm{U}$ a las de unidades.

La presente investigación se encuentra clasificada en la categoría de investigación sin riesgo, según el artículo 11 de la resolución 008430 de 1993, del Ministerio de Salud, debido a que la metodología aplicada consiste en hacer cálculos físicos sobre resultados de exámenes no invasivos de la práctica clínica que han sido previamente medicamente prescritos, haciendo innecesario el uso de consentimientos informados. También se protegió la privacidad, integridad y anonimato de los exámenes estudiados.

\section{Metodología Estadística}

Para el análisis estadístico, el diagnóstico convencional fue desenmascarado y se tomó como Gold-Estándar para compararlo con la metodología matemática. Se calculó especificidad y sensibilidad a través de una clasificación binaria, donde los verdaderos positivos (VP) corresponden al número de pacientes anormales de acuerdo al GoldEstándar y que se encuentran dentro de los valores matemáticos correspondientes a anormalidad, falsos positivos (FP) aquellos que matemáticamente se comportan como estudios anormales y cuyo diagnóstico clínico es normal, falsos negativos (FN) los que han sido diagnosticados matemáticamente como normales pero cuyos valores clínicos se corresponden con pacientes anormales, y finalmente verdaderos negativos (VN) definidos como el número de registros cardíacos diagnosticados clínicamente como normales y cuyos valores matemáticos también se corresponden con normalidad.

La concordancia entre los valores físico-matemáticos y el diagnóstico clínico convencional se calculó con el coeficiente Kappa a través de la siguiente fórmula:

$$
K=\frac{C o-C a}{T o-C a} \quad \text { Ecuación } 6
$$

Donde:

Co: número de concordancias observadas, es decir, número de pacientes con el mismo diagnóstico de acuerdo con la nueva metodología propuesta y con el Gold-Estándar.

To: totalidad de observaciones, es decir, la totalidad de casos normales y con enfermedad.

Ca: Concordancias atribuibles al azar, que se calculan de acuerdo con la siguiente fórmula

$$
C a=\left[\left(f_{1} \times C_{1}\right) / T o\right]+\left[\left(f_{2} \times C_{2}\right) / T o\right] \quad \text { Ecuación } 7
$$

Donde $\mathrm{f}_{1}$ es el número de pacientes que presentan valores matemáticos dentro de los límites de normalidad, $\mathrm{C}_{1}$ es el número de pacientes diagnosticados clínicamente dentro de la normalidad, $\mathrm{f}_{2}$ es el número de pacientes que presentan valores matemáticos asociados a anormalidad, $C_{2}$ es el número de pacientes diagnosticados clínicamente con alguna patología y $T_{0}$ es el número total de casos normales y anormales.

\section{Resultados}

Las parejas ordenadas en rangos de a cinco para las frecuencias de los atractores cardiacos variaron entre $[40,40]$ y $[165,165]$; entre $[55,55]$ y $[165,165]$ para las dinámicas normales y para las anormales entre $[40,40]$ y $[135,135]$. Se encontró que los valores de probabilidad de las frecuencias de ocupación de las parejas de la frecuencia cardíaca en el espacio de fases estuvieron entre 0 y 0,152 , con frecuencias de ocupación entre 0 y 8314 respectivamente. Se encontró que los valores de la entropía para los Holter anormales variaron entre $3,91 \times 10^{-23}$ y $6,80 \times 10^{-23} \mathrm{y}$, para los normales, entre $6,41 \times 10^{-23}$ y $7,24 \times 10^{-23}$ (datos no mostrados), confirmando los resultados de la metodología diagnóstica desarrollada previamente, donde los valores para los casos normales deberían ser mayores o iguales a $6,39 \times 10^{-23}$. De la misma forma, el máximo valor de entropía para los casos agudos fue de $4,86 \times 10^{-23}$, confirmando que 
las dinámicas agudas tendrían un valor menor o igual a $5,07 \times 10^{-23}(29)$.

El valor de las proporciones de la entropía se encontró entre 0 y 5,64 (Tabla 2), y para las sumas de las restas de las proporciones que se encontraron fuera de los límites de la normalidad, los valores estuvieron entre 0 y 3,70 (Tabla 3). Se evidenció que por lo menos dos de las proporciones evaluadas para los atractores anormales en cualquiera de las regiones no se encuentran contenidas dentro de los límites de normalidad, confirmando el parámetro diagnóstico encontrado previamente. Después de realizar las medidas físico-matemáticas y desenmascarar las conclusiones clínicas de los Holter se calcularon las medidas de especificidad y sensibilidad, siendo del 100\% cada una, con un coeficiente Kappa de 1, su máximo valor posible.

Tabla 2. Valores de proporciones de la entropía para cada región. Resultados para 28 de los Holter estudiados

\begin{tabular}{|c|c|c|c|c|c|c|c|c|c|c|c|c|c|c|c|c|c|c|}
\hline \multirow{2}{*}{$\mathrm{N}$} & \multicolumn{6}{|c|}{ REGION 1} & \multicolumn{6}{|c|}{ REGION 2} & \multicolumn{6}{|c|}{ REGION 3} \\
\hline & $\mathrm{U} / \mathrm{T}$ & D/T: & $\mathrm{C} / \mathrm{T}:$ & M/T: & $\mathrm{C} / \mathrm{M}:$ & D/C: & $\mathrm{U} / \mathrm{T}:$ & $\mathrm{D} / \mathrm{T}:$ & $\mathrm{C} / \mathrm{T}:$ & M/T: & $\mathrm{C} / \mathrm{M}:$ & D/C: & $\mathrm{U} / \mathrm{T}:$ & $\mathrm{D} / \mathrm{T}:$ & $\mathrm{C} / \mathrm{T}:$ & M/T: & C/M: & D/C: \\
\hline 1 & 0,0000 & 0,0223 & 0,3602 & 0,6066 & 0,5938 & 0,0620 & 0,0013 & 0,0096 & 0,0000 & 0,0000 & & & 0,0000 & 0,0000 & 0,0000 & 0,0000 & & \\
\hline 2 & 0,0002 & 0,0177 & 0,3905 & 0,5805 & 0,6726 & 0,0453 & 0,0000 & 0,0005 & 0,0106 & 0,0000 & & 0,0509 & 0,0000 & 0,0000 & 0,0000 & 0,0000 & & \\
\hline 3 & 0,0000 & 0,0221 & 0,1993 & 0,7755 & 0,2570 & 0,1108 & 0,0000 & 0,0030 & 0,0000 & 0,0000 & & & 0,0000 & 0,0000 & 0,0000 & 0,0000 & & \\
\hline 5 & 0,0006 & 0,0764 & 0,1464 & 0,5444 & 0,2689 & 0,5220 & 0,0002 & 0,1731 & 0,0399 & 0,0000 & & 4,3443 & 0,0005 & 0,0184 & 0,0000 & 0,0000 & & \\
\hline 6 & 0,0000 & 0,0000 & 0,2165 & 0,2585 & 0,8376 & 0,0000 & 0,0000 & 0,0000 & 0,0081 & 0,3135 & 0,0257 & 0,0000 & 0,0006 & 0,0137 & 0,1893 & 0,0000 & & 0,0721 \\
\hline 7 & 0,0002 & 0,0903 & 0,4944 & 0,3145 & 1,5721 & 0,1826 & 0,0050 & 0,0682 & 0,0275 & 0,0000 & & 2,4817 & 0,0000 & 0,0000 & 0,0000 & 0,0000 & & \\
\hline 8 & 0,0000 & 0,0754 & 0,4884 & 0,3402 & 1,4355 & 0,1543 & 0,0058 & 0,0664 & 0,0238 & 0,0000 & & 2,7933 & 0,0000 & 0,0000 & 0,0000 & 0,0000 & & \\
\hline 10 & 0,0000 & 0,0454 & 0,3917 & 0,3974 & 0,9857 & 0,1158 & 0,0011 & 0,1343 & 0,0000 & 0,0000 & & & 0,0028 & 0,0274 & 0,0000 & 0,0000 & & \\
\hline 11 & 0,0000 & 0,0332 & 0,3502 & 0,6037 & 0,5801 & 0,0948 & 0,0000 & 0,0066 & 0,0063 & 0,0000 & & 1,0521 & 0,0000 & 0,0000 & 0,0000 & 0,0000 & & \\
\hline 12 & 0,0002 & 0,0904 & 0,5049 & 0,3045 & 1,6583 & 0,1790 & 0,0050 & 0,0677 & 0,0273 & 0,0000 & & 2,4821 & 0,0000 & 0,0000 & 0,0000 & 0,0000 & & \\
\hline 13 & 0,0006 & 0,0040 & 0,2220 & 0,2369 & 0,9371 & 0,0180 & 0,0005 & 0,0392 & 0,0096 & 0,2916 & 0,0330 & 4,0672 & 0,0007 & 0,0122 & 0,1012 & 0,0814 & 1,2435 & 0,1210 \\
\hline 14 & 0,0000 & 0,0173 & 0,3263 & 0,6495 & 0,5023 & 0,0531 & 0,0002 & 0,0067 & 0,0000 & 0,0000 & & & 0,0000 & 0,0000 & 0,0000 & 0,0000 & & \\
\hline 15 & 0,0003 & 0,0310 & 0,2735 & 0,6911 & 0,3958 & 0,1134 & 0,0001 & 0,0039 & 0,0000 & 0,0000 & & & 0,0000 & 0,0000 & 0,0000 & 0,0000 & & \\
\hline 16 & 0,0019 & 0,0115 & 0,3147 & 0,6512 & 0,4832 & 0,0366 & 0,0006 & 0,0170 & 0,0030 & 0,0000 & & 5,6445 & 0,0000 & 0,0000 & 0,0000 & 0,0000 & & \\
\hline 17 & 0,0000 & 0,0485 & 0,5142 & 0,3459 & 1,4866 & 0,0943 & 0,0035 & 0,0632 & 0,0248 & 0,0000 & & 2,5426 & 0,0000 & 0,0000 & 0,0000 & 0,0000 & & \\
\hline 18 & 0,0006 & 0,0353 & 0,2379 & 0,7215 & 0,3298 & 0,1484 & 0,0002 & 0,0045 & 0,0000 & 0,0000 & & & 0,0000 & 0,0000 & 0,0000 & 0,0000 & & \\
\hline 20 & 0,0000 & 0,0794 & 0,2906 & 0,6098 & 0,4765 & 0,2731 & 0,0002 & 0,0094 & 0,0105 & 0,0000 & & 0,8968 & 0,0000 & 0,0000 & 0,0000 & 0,0000 & & \\
\hline 21 & 0,0004 & 0,0112 & 0,2020 & 0,3360 & 0,6012 & 0,0552 & 0,0000 & 0,0019 & 0,0006 & 0,2896 & 0,0020 & 3,3714 & 0,0000 & 0,0072 & 0,1513 & 0,0000 & & 0,0475 \\
\hline 22 & 0,0000 & 0,0430 & 0,2817 & 0,6688 & 0,4213 & 0,1526 & 0,0000 & 0,0018 & 0,0046 & 0,0000 & & 0,3968 & 0,0000 & 0,0000 & 0,0000 & 0,0000 & & \\
\hline 23 & 0,0000 & 0,0271 & 0,2377 & 0,7155 & 0,3322 & 0,1140 & 0,0003 & 0,0083 & 0,0111 & 0,0000 & & 0,7454 & 0,0000 & 0,0000 & 0,0000 & 0,0000 & & \\
\hline 24 & 0,0022 & 0,0167 & 0,1841 & 0,7813 & 0,2357 & 0,0910 & 0,0000 & 0,0051 & 0,0105 & 0,0000 & & 0,4885 & 0,0000 & 0,0000 & 0,0000 & 0,0000 & & \\
\hline 25 & 0,0019 & 0,0868 & 0,3285 & 0,4952 & 0,6635 & 0,2643 & 0,0013 & 0,0341 & 0,0094 & 0,0270 & 0,3470 & 3,6427 & 0,0003 & 0,0156 & 0,0000 & 0,0000 & & \\
\hline 26 & 0,0000 & 0,0392 & 0,2831 & 0,6494 & 0,4359 & 0,1383 & 0,0006 & 0,0150 & 0,0127 & 0,0000 & & 1,1774 & 0,0000 & 0,0000 & 0,0000 & 0,0000 & & \\
\hline 27 & 0,0000 & 0,0348 & 0,2685 & 0,6819 & 0,3937 & 0,1296 & 0,0007 & 0,0052 & 0,0089 & 0,0000 & & 0,5909 & 0,0000 & 0,0000 & 0,0000 & 0,0000 & & \\
\hline 28 & 0,0000 & 0,0764 & 0,5178 & 0,3054 & 1,6956 & 0,1476 & 0,0037 & 0,0697 & 0,0269 & 0,0000 & & 2,5878 & 0,0000 & 0,0000 & 0,0000 & 0,0000 & & \\
\hline
\end{tabular}


Tabla 3. Valores de entropía, relación S/ky sumas de las restas de los valores por fuera de los límites de normalidad, respecto a estos límites.

\begin{tabular}{|c|c|c|c|c|c|c|}
\hline \multirow{2}{*}{$\mathbf{N}$} & \multirow{2}{*}{$\mathrm{S} / \mathrm{k}$} & \multirow{2}{*}{ Entropía } & \multicolumn{4}{|c|}{ SUMAS } \\
\hline & & & $\mathrm{U}$ & D & C & M \\
\hline 1 & $-4,1903$ & 5,7827E-23 & 0,00 & 0,02 & 0,14 & 0,55 \\
\hline 2 & $-4,0875$ & $5,6408 \mathrm{E}-23$ & 0,00 & 0,02 & 2,54 & 0,44 \\
\hline 3 & $-3,5954$ & $4,9617 \mathrm{E}-23$ & 0,00 & 0,02 & 0,28 & 1,05 \\
\hline 4 & $-4,1298$ & $5,6991 \mathrm{E}-23$ & 0,00 & 0,01 & 2,49 & 0,79 \\
\hline 5 & $-4,1102$ & $5,6721 \mathrm{E}-23$ & 0,00 & 0,06 & 1,71 & 0,81 \\
\hline 6 & $-3,6256$ & $5,0033 \mathrm{E}-23$ & 0,00 & 0,05 & 3,07 & 2,50 \\
\hline 7 & $-4,6445$ & $6,41 \mathrm{E}-23$ & 0,00 & 0,00 & 0,00 & 0,00 \\
\hline 8 & $-4,9790$ & 6,8711E-23 & 0,00 & 0,00 & 0,00 & 0,00 \\
\hline 9 & $-4,1815$ & $5,7705 \mathrm{E}-23$ & 0,00 & 0,00 & 1,83 & 0,87 \\
\hline 10 & $-4,7777$ & 6,5932E-23 & 0,00 & 0,03 & 0,09 & 0,03 \\
\hline 11 & $-4,1482$ & $5,7246 \mathrm{E}-23$ & 0,00 & 0,01 & 1,54 & 0,56 \\
\hline 12 & $-4,6985$ & $6,4839 \mathrm{E}-23$ & 0,00 & 0,00 & 0,00 & 0,00 \\
\hline 13 & $-3,7792$ & $5,2154 \mathrm{E}-23$ & 0,00 & 0,05 & 1,31 & 3,70 \\
\hline 14 & $-4,2172$ & 5,8197E-23 & 0,00 & 0,02 & 0,18 & 0,68 \\
\hline 15 & $-4,2642$ & $5,8846 \mathrm{E}-23$ & 0,00 & 0,01 & 0,21 & 0,83 \\
\hline 16 & $-4,3515$ & $6,0050 \mathrm{E}-23$ & 0,00 & 0,03 & 2,56 & 0,70 \\
\hline 17 & $-5,2185$ & $7,2015 \mathrm{E}-23$ & 0,00 & 0,00 & 0,00 & 0,00 \\
\hline 18 & $-3,4772$ & $4,7985 \mathrm{E}-23$ & 0,00 & 0,00 & 0,24 & 0,93 \\
\hline 19 & $-5,0005$ & $6,9007 \mathrm{E}-23$ & 0,00 & 0,02 & 1,45 & 0,26 \\
\hline 20 & $-4,3236$ & $5,9666 \mathrm{E}-23$ & 0,00 & 0,00 & 1,84 & 0,67 \\
\hline 21 & $-3,5285$ & 4,8693E-23 & 0,00 & 0,03 & 0,57 & 2,74 \\
\hline 22 & $-4,1869$ & 5,7779E-23 & 0,00 & 0,00 & 2,26 & 0,78 \\
\hline 23 & $-4,0244$ & $5,5537 \mathrm{E}-23$ & 0,00 & 0,01 & 1,96 & 0,92 \\
\hline 24 & $-11,5302$ & 1,5911E-22 & 0,00 & 0,02 & 2,27 & 1,08 \\
\hline 25 & $-4,4175$ & $6,0962 \mathrm{E}-23$ & 0,00 & 0,02 & 0,57 & 2,09 \\
\hline 26 & $-4,2545$ & $5,8712 \mathrm{E}-23$ & 0,00 & 0,00 & 1,48 & 0,75 \\
\hline 27 & $-4,3707$ & $6,0316 \mathrm{E}-23$ & 0,00 & 0,00 & 2,08 & 0,82 \\
\hline 28 & $-5,1754$ & $7,1421 \mathrm{E}-23$ & 0,00 & 0,00 & 0,00 & 0,00 \\
\hline
\end{tabular}

Al observar las conclusiones clínicas de los Holters se observó que varios de ellos correspondían a un mismo paciente en fechas diferentes. Así por ejemplo, los Holters 6, 9 y 26 (Tabla 3), corresponden a un paciente que fue internado en la unidad de cuidados coronarios por presentar infarto agudo de miocardio (IAM). La dinámica del día en que lo internaron (Holter 6) presenta un valor de miles de 2,50 , que es un valor característico de enfermedad aguda.
Dos días después (Holter 9) y luego una semana después (Holter 26), se volvieron a tomar Holters, observando que los valores de miles disminuyeron para el Holter 9, siendo 0,87, y luego siguieron disminuyendo para el Holter 26, siendo de 0,75. Esto mostró matemáticamente que el paciente evolucionaba hacia la normalidad y que el tratamiento seguido había sido exitoso (Tabla 4), lo que fue corroborado con la evolución clínica. 
Tabla 4. Evolución del paciente. Se observan las sumas de las restas agrupadas de acuerdo a la metodología aplicada respecto a estos límites.

\begin{tabular}{cccccc}
$\begin{array}{c}\text { Holter } \\
\text { No. }\end{array}$ & Día & M & C & D & U \\
\hline $\mathbf{6}$ & 1 & 2,50 & 3,07 & 0,05 & 0,00 \\
$\mathbf{9}$ & 3 & 0,87 & 1,83 & 0,00 & 0,00 \\
$\mathbf{2 6}$ & 11 & 0,75 & 1,48 & 0,00 & 0,00 \\
\hline
\end{tabular}

Otro caso que también se pudo observar fue el de un paciente que ingresó con enfermedad coronaria severa fue sometido a cateterismo cardíaco y colocación de Stent, los holters 18, 23 y 3 (Tabla 3) corresponden a la dinámica del paciente; el primer registro (Holter 18) corresponde a la dinámica del paciente post operatorio de colocación del stent y presentaba unos valores de miles de 0,93 , al siguiente dia se le realizó otro monitoreo (Holter 23) que mostró una disminución en los valores de miles 0,92, mostrando una mejoría en el paciente, lo que se corroboró con la historia clínica, en la que el paciente no reportaba ningún dolor ni síntoma desfavorable, sin embargo en el tercer monitoreo (Holter 3 ) los valores de miles ascendieron a 1,05, mostrando un evento agudo, lo que se correlaciona con el reporte de IAM del paciente (Tabla 5).

Tabla 5. Evolución del paciente sometido a cateterismo cardiaco y colocación de stent. Se observan las sumas de las restas agrupadas de acuerdo a la metodología aplicada, véanse la disminución y luego el aumento abrupto de los valores de miles (M).

\begin{tabular}{cccccc}
$\begin{array}{c}\text { Holter } \\
\text { No. }\end{array}$ & Día & M & C & D & U \\
\hline $\mathbf{1 8}$ & 1 & 0,93 & 0,24 & 0,00 & 0,00 \\
23 & 2 & 0,92 & 1,96 & 0,01 & 0,00 \\
$\mathbf{3}$ & 3 & 1,05 & 0,28 & 0,02 & 0,00 \\
\hline
\end{tabular}

\section{Discusión}

Este es el primer trabajo en el que se confirma, en una población de 400 Holters, la aplicación clínica de la metodología desarrollada a partir del cálculo de las proporciones de la entropía y el análisis de aparición de pares ordenados dinámicos de los atractores caóticos en el espacio de fases, confirmando su capacidad diagnóstica para establecer diferencias entre normalidad, anormalidad y evolución entre éstas. Los resultados obtenidos demuestran la capacidad predictiva y diagnóstica de la teoría desarrollada, así como su aplicabilidad clínica a cada caso particular sin necesidad de los parámetros poblacionales de la epidemiología. Esta metodología, al basarse en el orden geométrico y matemático del espacio universal de los atractores caóticos, desarrolla predicciones matemáticas que no necesitan validación estadística. El análisis estadístico realizado en este artículo se hizo como parte de los requisitos de la literatura médica actual.
La aplicación de teorías matemáticas y físicas al registro Holter (12-23), en contraste con el análisis de la variabilidad de la frecuencia cardíaca a partir de los cambios RR, ha revaluado el concepto de variabilidad como parámetro diagnóstico y predictor de la dinámica cardíaca. Se ha mostrado que la dinámica cardíaca presenta un comportamiento caótico o irregular (38), contrario a la concepción homeostática, cuyo diagnóstico de normalidad y enfermedad corresponden a regularidad $e$ irregularidad respectivamente. Gold Berger y cols. (12) aplicando la teoría de sistemas dinámicos desarrollaron una concepción de salud - enfermedad aplicada a la fisiología cardíaca, donde la normalidad se encuentra en el rango intermedio entre la extrema irregularidad y la extrema regularidad, que corresponden al comportamiento patológico. También se desarrollaron medidas predictivas de mortalidad con dimensiones fractales de la frecuencia cardíaca en pacientes diagnosticados con infarto agudo de miocardio (IAM) con fracción de eyección (FE) menor a 35\%, encontrando factores predictores de muerte más confiables (13).

La aplicación de métodos de dinámica no lineal ha mostrado nuevas ideas acerca del comportamiento de la dinámica de la frecuencia cardíaca, así como también ha definido diferentes índices para el estudio de dicha dinámica. Sin embargo, la aplicabilidad de estas metodologías debe ser sometida a la realización de más estudios (24) que permitan aclarar qué métodos deben ser aplicados bajo ciertas condiciones estandarizadas (25). También se ha discutido la capacidad de las técnicas convencionales de análisis no lineal de series de tiempo en el estudio de la dinámica cardíaca, pues éstas generalmente carecen de sensibilidad y especificidad suficientes para discriminar una dinámica caótica del ruido aleatorio (39), así como de métodos basados en medidas fractales o entrópicas dado que han mostrado ser indicadores pobres de la no linealidad de la dinámica cardíaca (40).

Esta metodología está fundamentada en teorías físicas y matemáticas, (sistemas dinámicos y probabilidad) y en leyes y principios de la física teórica (probabilidad y entropía) lo que permite que la metodología sea aplicable a cada caso particular en la práctica clínica diaria como un método de ayuda diagnóstica, de prevención y de evaluación de intervenciones, pues no requiere para su aplicación de metodologías epidemiológicas que dependan de poblaciones. El concepto de entropía fue definido originalmente por Carnot (41) en la teoría termodinámica, luego fue generalizado en la mecánica estadística $(34,35)$, posteriormente fue reinterpretado (36-39,41, 42). En este trabajo la entropía es una medida del orden subyacente del atractor geométrico que muestra la auto organización de las distribuciones de probabilidades, que permite un análisis teórico de la totalidad del fenómeno. 
Analógicamente los atractores numéricos se pueden observar como ventanas temporales (43) definidos en un espacio finito y acotado que permiten establecer estados geométricos intrínsecos que sustentan las diferencias numéricas entre normalidad y anormalidad. La evolución de la dinámica cardíaca se obtiene con la sucesión de ventanas temporales como se observó en los casos en los que diferentes Holters correspondían a un mismo paciente pero en diferentes tiempos. En estos casos la metodología mostró la capacidad de evaluación y de predicción de la evolución del paciente, evidenciando los cambios en la dinámica en los valores numéricos obtenidos, permitiendo que por ejemplo en el segundo caso (tabla 5) aunque el paciente no presentaba síntomas que mostraran que la intervención no había sido exitosa, las medidas matemáticas sí lo evidenciaron notablemente con un aumento en los valores de miles, lo que se corroboró clínicamente pues el paciente presentó posteriormente un IAM. Este tipo de resultados en los que se ha predicho matemáticamente la evolución de un paciente también se han obtenido en otros trabajos (44), lo que sugiere que las predicciones obtenidas son de gran ayuda a nivel clínico para evaluar la evolución en la recuperación de pacientes que han sido intervenidos farmacológica y/o quirúrgicamente.

La concepción causal-determinista de la física clásica fue revaluada con la aparición de la mecánica estadística, la mecánica cuántica y la teoría del caos, en las que no necesariamente existe una relación causal entre los fenómenos, y no se puede establecer en forma determinista la evolución de los mismos. Sin embargo, la indeterminación o aleatoriedad, así como la determinación, son necesarias para la física (45). En este trabajo se observa la dinámica cardíaca desde leyes y teorías de la física, pero desde una posición determinista-indeterminista en donde se observa el atractor como un sistema de auto organización simultánea no equiprobable de los rangos de frecuencias. Así, habrá rangos más probables que otros y la probabilidad estará "cargada" en lugares particulares del atractor (46). La aplicabilidad de esta concepción en la caracterización de una dinámica considerada caótica sugiere que el orden encontrado puede sustentar un principio general para cualquier sistema dinámico caótico.

\section{Agradecimientos}

A la Universidad Militar Nueva Granada, especialmente a Vicerrectoría de Investigaciones, al Fondo de Investigaciones y a la Facultad de Medicina por apoyar nuestros trabajos. Agradecemos especialmente a las Doctoras Jacqueline Blanco, Vicerrectora de Investigaciones, Martha Bahamón, Vicerrectora Académica, Esperanza Fajardo, Directora de Investigaciones de la Facultad de Medicina, y a los Doctores Juan Miguel Estrada, Decano de la Facultad de
Medicina, Alejandro Castro, Jefe de la División de Investigación Científica, y a Henry Acuña, por su apoyo a nuestras investigaciones.

\section{Conflictos de interés}

Los autores declaran no tener de manera directa o indirecta, ningún tipo de conflicto de intereses financieros, académicos o laborales que puedan poner en peligro la validez de este estudio.

\section{Financiación}

Producto derivado del proyecto MED-1078 financiado por el Fondo de Investigaciones Universidad Militar Nueva Granada.

\section{Dedicatoria}

A nuestros hijos.

\section{Referencias}

1. OMS - Centro de prensa. Enfermedades Cardiovasculares Nota descriptiva. [Internet] marzo de 2013. [acceso abril 13 de 2013]. Disponible en http://www.who.int/mediacentre/ factsheets/fs317/es/index.html.

2. Robledo R, Escobar FA. Las enfermedades crónicas no transmisibles en Colombia. Boletín del Observatorio en Salud. 2010;3(4):1-9.

3. Devaney R. A first course in chaotic dynamical systems theory and experiments. Reading Mass.: Addison- Wesley; 1992.p.148.

4. Mandelbrot B. The fractal geometry of nature. Tusquets Eds S.A. Barcelona; 2000. p. 341-348.

5. Peitgen H, Jurgens H, Saupe D. Chaos and Fractals: New Frontiers of Science. New York: Springer-Verlag; 1992.p.67766.

6. Rodríguez J. Comportamiento fractal del repertorio $\mathrm{T}$ específico contra el alérgeno Poa P9. Rev Fac Med Univ Nac Colomb. 2005;53(2):72-78.

7. Burgos J. Fractal representation of the immune B cell repertoire. Bio Systems. 1996; 39:19- 24.

8. Peitgen, H. Length area and dimension. Measuring complexity and scalling properties. Chaos and Fractals: New Frontiers of Science. New York: Springer-Verlag; 1992.p.183-228.

9. Bassingthwaighte JB, Liebovitch LS, West BJ. Fractal Physiology. Oxford Univ. Press.N.Y.; 1994.

10. Goldberger A, West BJ. Fractals in physiology and medicine. Yale Journal of Biology. 1987; 60:421-435.

11. West BJ. Fractal Physiology and Chaos Medicine. London. World Scientific Publishing, Co., 1990.

12. Goldberger A, Amaral L, Hausdorff J, Ivanov P, Peng C, Stanley H. Fractal dynamics in physiology: alterations with disease and aging. Proc Natl Acad Sci USA. 2002;99(suppl1): 2466-72.

13. Huikuri HV, Mäkikallio T, Peng CK, Goldberger A, Hintze U, Moller M. Fractal correlation properties of R-R interval dynamics and mortality in patients with depressed left ventricular function after and acute myocardial infarction. Circulation. 2000;101:47-53.

14. Richman JS, Moorman JR. Physiological time-series analysis 
using approximate entropy and sample entropy. Am J Physiol Heart Circ Physiol 2000;278:H2039-H2049.

15. Peng CK, Havlin S, Stanley HE, Goldberger AL. Quantification of scaling exponents and crossover phenomena in nonstationary heartbeat time series. Chaos 1995;5: 82-87.

16. Mäkikallio TH, Huikuri HV, Hintze U, Videbaek J, Mitrani RD, Castellanos A, et al. Fractal analysis and time and frequencydomain measures of heart rate variability as predictors of mortality in patients with heart failure. Am J Cardiol. 2001;87(2):178-182.

17. Porta A, Guzzetti S, Montano N, Furlan R, Pagani M, Malliani A, Cerutti S. Entropy, entropy rate and pattern classification as tools to typify complexity in short heart period variability series. IEEE Trans. Biomed Eng. 2001;48:1282-1291.

18. Guzzetti S, Borroni E, Garbelli PE, Ceriani E, Della Bella P, Montano N, et al. Symbolic dynamics of heart rate variability: a probe to investigate cardiac autonomic modulation. Circulation. 2005; 112:465-470.

19. Maestri R, Pinna GD, Accardo A, Allegrini P, Balocchi R, D'Addio G, et al. Nonlinear indices of heart rate variability in chronic heart failure patients: redundancy and comparative clinical value. J. Cardiovasc. Electrophysiol. 2007; 18:425-433.

20. Costa M, Goldberger AL, Peng CK. Multiscale entropy analysis of biological signals. Phys Rev E 2005;71(Pt 1):021 906.

21. Baselli G, Porta A, Pagani M. Coupling arterial windkessel with peripheral vasomotion: modeling the effects on lowfrequency oscillations. IEEE Trans Biomed. Eng. 2006;53:53-64.

22. Khoo MC. Modeling of autonomic control in sleep-disordered breathing. Cardiovasc. Eng. 2008;8:30-41.

23. Ivanov PC, Amaral LAN, Goldberger AL, Havlin S, Rosenblum MG, Struzik ZHE, et al. Multifractality in human heartbeat dynamics. Nature. 1999; 399:461-465.

24. Perkiömäki J, Mäkikallio TH, Huikuri HV. Fractal and Complexity Measures of Heart Rate Variability. Clin. Exp. Hypertens. 2005;27(2-3):149-158.

25. Voss A, Schulz S, Schroeder R, Baumert M, Caminal P. Methods derived from nonlinear dynamics for analysing heart rate variability. Phil Trans R Soc 2009; 367(1887):277-296.

26. Rodríguez J. Dynamical systems theory and ZIPF - Mandelbrot law applied to the development of a fetal monitoring diagnostic methodology. XVIII FIGO World Congress of Gynecology And Obstetric. Kuala Lumpur, Malaysia. November 2006.

27. Rodríguez J, Prieto S, Avilán N, Correa C, Bernal P, Ortiz L, et al. Nueva metodología física y matemática de evaluación del Holter Rev Colomb Cardiol 2008; 15:50-54.

28. Rodríguez J, Correa C, Ortiz L, Prieto S, Bernal P, Ayala J. Evaluación matemática de la dinámica cardíaca con la teoría de la probabilidad. Rev Mex Cardiol. 2009;20 (4):183-189.

29. Rodríguez J. Entropía proporcional de los sistemas dinámicos cardiacos: predicciones físicas y matemáticas de la dinámica cardíaca de aplicación clínica. Rev Colomb Cardiol 2010;17:115-129.

30. Rodríguez J, Prieto S, Bernal P, Izasa D, Salazar G, Correa C, et al. Entropía proporcional aplicada a la evolución de la dinámica cardíaca. Predicciones de aplicación clínica. La emergencia de los enfoques de la complejidad en América Latina. Tomo I. Comunidad Editora Latinoamericana. Argentina; 2015.p.247-264.

31. Rodríguez J, Proportional Entropy of the cardiac dynamics in $\mathrm{CCU}$ patients. 7th International Meeting Intensive Cardiac Care. Israel Heart Society, European Society of Cardiology, EBAC: Tel Aviv, Israel, 2011.

32. Pineda M, Matiz H, Rozo R. Enfermedad coronaria. Bogotá: Editorial Kimpres Ltda; 2002.

33. Kolmogorov AN. Foundations of the theory of probability. New York: Chelsea Publishing Company; 1950.
34. Feynman RP, Leighton RB, Sands M. Probabilidad. Física. Vol. 1. Primera edición México: Addison-Wesley Iberoamericana, S. A.;1998.p.1-16.

35. Matvéev A. Física molecular. Primera edición, MIR,Moscú; 1987.

36. Tolman R. Principles of statistical mechanics. Primera edición. New York: Dover Publications; 1979.

37. Machta J. Entropy, information, and computation. Am J Phys 1999;67(12),1074-1077.

38. Denton TA, Diamond GA, Helfant RH, Khan S, Karagueuzian $\mathrm{H}$. Fascinating rhythm: A primer on chaos theory and its application to cardiology. American Heart Journal 1990:120(6);1419-40

39. Braun C, Kowallik P, Freking A, Hadeler D, Kniffki K, Meesmann M. Demonstration of nonlinear components in heart rate variability of healthy persons Am J Physiol 1998;275:H1577-H1584.

40. Wu GQ, Arzeno NM, Shen LL, Tang DK, Zheng DA, Zhao NQ, et al. Chaotic Signatures of Heart Rate Variability and Its Power Spectrum in Health, Aging and Heart Failure. PLoS ONE 2009;4(2):e4323.

41. Cheng L, Guang H, Gou W, Chi P. Fractal, entropic and chaotic approaches to complex physiological time series analysis: A critical appraisal. 31st Annual International Conference of the IEEE EMBS Minneapolis, Minnesota, USA. 2009; 3429-3432.

42. Feynman RP, Leighton RB, Sands M.. Leyes de la Termodinámica. Física. Vol. 1. Primera edición Addison-Wesley Iberoamericana, S.A. México;1998.p.1-19.

43. Bekenstein J. La información en el Universo holográfico. Investigación y ciencia: Octubre 2003;p.36-43.

44. Rodríguez J, Prieto S, Domínguez D, Melo M, Mendoza F, Correa C,et al. Mathematical-physical prediction of cardiac dynamics using the proportional entropy of dynamic systems. J. Med. Med. Sci. 2013; 4(8): 370-381.

45. Fernández-Rañada A. Introducción. Orden y Caos. Scientific American. 1990. Prensa Científica S.A.; p. 4-8.

46. Crutchfield JP. Between order and chaos. Nat Phys. 2012;8:17-24. 